\title{
Basic rheology of dermal filler
}

\author{
Moon Seop Choi \\ Grace Plastic Surgery Clinic, Seoul, Korea
}

Dermal injectables are the most popular material for facial enhancement, including volumizing and wrinkle correction. However, although hyaluronic acid is the most common component of dermal injectables, the character of hyaluronic acid products is quite variable. These materials can be described in terms of their viscoelastic properties using four parameters. In this article, the author would like to describe these properties in detail. Although many physicians consider this material to be convoluted, thoroughly understanding the characteristics of various injectables enables us to tailor procedures accordingly and to enhance the final results.

Keywords Injectables / Filler / Rheology / Hyaluronic acid

Correspondence: Moon Seop Choi Grace Plastic Surgery Clinic, 623 Gangnam-daero, Seocho-gu, Seoul 06524, Korea

Tel: +82-2-555-8558

Fax: + 82-2-556-7582

E-mail:mschoi@graceclinic.co.kr

This review article was prepared by the Botulinum Toxin-Filler-Thread Academic Association for the Korean Plastic \& Reconstructive Surgeon.

Received: April 24, 2020 • Revised: May 19, 2020 • Accepted: June 22, 2020

pISSN: 2234-6163 • elSSN: 2234-6171・ https://doi.org/10.5999/aps.2020.00731 • Arch Plast Surg 2020;47:301-304

\section{INTRODUCTION}

Many injectable dermal fillers have been developed in recent years, most of which contain hyaluronic acid (HA). These fillers are commonly used for wrinkle correction or volumizing, especially in the forehead, nose, nasolabial fold, chin, and infraorbital area $[1,2]$.

For over 20 years, bovine and human collagen material was used in injectables with approval from the United States Food and Drug Administration. In December 2003, Restylane (QMed AB, Uppsala, Sweden) received approval in the United States and entered the filler market. Restylane has various particle sizes, upon which the characteristics of the material depend. In the early 2000s, many researchers started to use the word "biphasic" to describe particle-containing fillers, and the "monophasic" type was also developed. A representative example of a mono-phasic material is Juvéderm (Allergan Inc., Irvine, CA, USA), which has no particles and is controlled by the degree of cross-linking. With high cross-linking, the filler is hard and is suitable for volumizing. However, the terms "bi-phasic," "monophasic," "size of particle," and "degree of cross-linking" only de- scribe the characteristics of fillers to a limited extent, and these more nuanced characteristics also contribute to the aesthetic results $[3,4]$.

Instead, to maximize outcomes, it is necessary to have a deep understanding of the properties of various fillers. However, the rheologic properties of fillers can be difficult to grasp. Although some articles have described these properties, the authors would like to explain them in simple and accessible terms to contribute to a broader understanding of this topic among plastic surgeons [5-10].

\section{HYALURONIC ACID}

HA is a polysaccharide that consists of D-glucuronic acid and D$\mathrm{N}$-acetylglucosamine disaccharide units (Fig. 1). These disaccharide units are termed monomers, and the small molecules that are formed by bonding between identical or different monomers are referred to as polymers. In HA polymers, units are linked to each other to form a linear chain through $\beta-1,4$ glycosidic bonds. The weight of each monomer is approximately $400 \mathrm{Da}$, and the polymerized chains can reach about $25 \mathrm{kDa}$. 
HA is an essential component of the extracellular matrix and exists in tissues as a biopolymer [8]. The basic unit of HA is the same regardless of its origin (e.g., animal or bacterial), but its length differs.

\section{MECHANICAL PROPERTIES}

Before filler is injected into the target tissue, it is important to consider shear deformation and compression/stretching forces as factors that can affect each other inside tissue through a complex interplay. Shear deformation occurs when a force is applied along the surface without a vertical vector, resulting in lateral shearing or torsion on the surface, while compression/stretching occurs when a force is applied vertically, as in compression or stretching (Fig. 2).

\section{Viscoelasticity}

Viscoelasticity refers to viscosity and elasticity when shear deformation occurs in an HA filler. Imagining a "gummy bear" jelly is a vivid analogy that can help us understand this property. Elasticity is defined as the degree of recovery when a shear force

\section{Fig. 1. Hyaluronic acid monomer unit}

Hyaluronic acid is a polysaccharide that consists of D-glucuronic (left) acid and D-N-acetylglucosamine (right) disaccharide units. They are bound together by a $\beta-1,3$ glycosidic bond. Additionally, two disaccharide units are linked by a $\beta-1,4$ glycosidic bond.

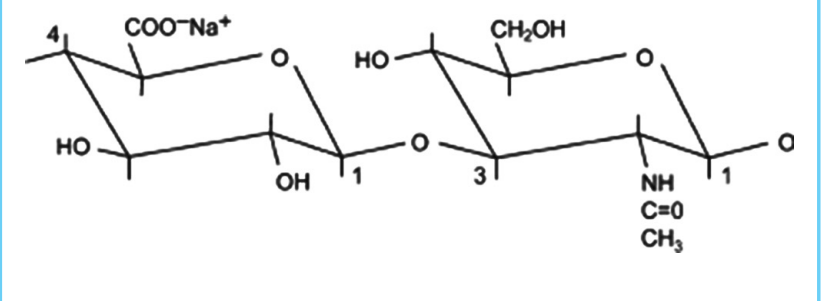

\section{Fig. 2. Two types of deformation}

Lateral shearing and torsion (left) and stretching and compression (right) [7].

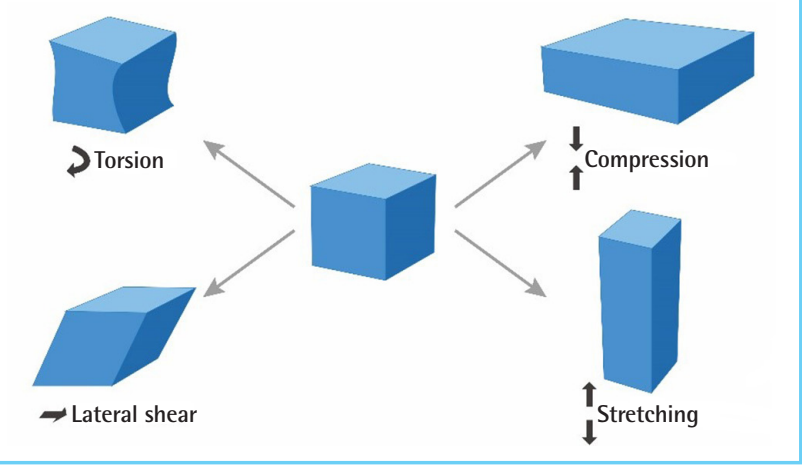

occurs and is then removed. Therefore, a stronger shear force is required to deform a material with high elasticity. On the contrary, if a material has high viscosity, such as honey, its degree of restoration after experiencing a shear force will be low.

Four rheological parameters are used to describe viscoelastic properties: $G^{*}$ (overall viscoelastic properties), G' (elastic properties), G" (viscous properties), and $\tan \delta$ (the ratio between viscous and elastic properties).

$\mathrm{G}^{*}$ is the complex modulus of the total energy that is needed to deform a material with shear force. It also refers to the "hardness" of multiple units of injectable filler, not the hardness of the entire deposited gel. Its formula is $\left|G^{*}\right|=\sqrt{\left(G^{\prime}\right)^{2}+\left(G^{\prime \prime}\right)^{2}}[7]$.

$\mathrm{G}^{\prime}$ is the elastic modulus of the energy fraction that is restored by the original shape after deformation. It represents the ability of a material to recover its shape after shear deformation. For example, if a "gummy bear" has high elasticity, it can recover its shape completely after a shear force is first applied and then removed. From a practical standpoint, $G^{\prime}$ is correlated with $G^{*}$.

G” is the viscous modulus of the energy fraction that is lost under shear deformation. G" reflects the inability of the gel to recover its original shape after a shear force is removed. Clinically, $G$ ” is related to injectability.

Tan $\delta$ is simply the ratio G" $/ G^{\prime}$, which indicates the elasticity of a material. If an injectable has a $\tan \delta<1$, it functions as a material with high elasticity, but if $\tan \delta>1$, it is similar to a viscous liquid such as honey. Clinically, a lower $\tan \delta$ is related with a high G', because HA fillers always have a low G”.

The viscoelastic properties of fillers are determined by the manufacturing process and design. The injectable fillers on the market have several types under the same brand name. From a practical standpoint, these properties related to the level of crosslinking or particle size.

\section{Cohesivity}

Cohesivity is described as the internal adhesion forces among

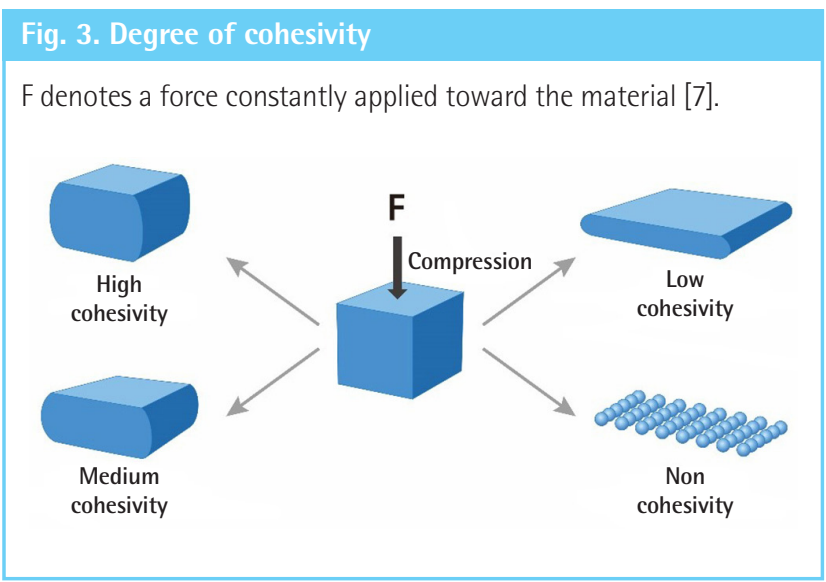


individual cross-linked HA units within an HA gel, as if the units are grasping each other [11]. Cohesivity can be measured by resistance after undergoing compression or vertical stretching (Fig. 3). It depends on the concentration of HA and some specific steps in the cross-linking process, but is not related to the level of cross-linking. A highly cohesive gel has a higher molding capacity just after injection, but less cohesive gels are more flexible. A previous study has suggested a novel method to measure cohesivity [9]. In that article, the authors proposed a semiquantitative method, with the following steps: dyeing of the filler, extrusion of the colored gel into a beaker of sterile water stirred at a constant rate, and an observation of how much and how fast the gel separates into particles. These results could be measured using a cohesivity scale.

\section{RHEOLOGIC APPLICATIONS FOR FACIAL INJECTION}

\section{Forehead}

There are two purposes of filler injections in the forehead: volumizing and fixation for fine wrinkles. For volumizing, a filler with a relatively high $G$ ' is needed because the filler must lift up the forehead soft tissue, and the extent of deformation should be lower. In contrast, fine wrinkles need meticulous control using a fine filler. In order to improve transverse wrinkles on the forehead, a botulinum toxin injection is requested in most patients who do not have severe blepharoptosis before the filler injection. The filler should have a smooth behavior and spread under the surface easily with a low $G$ ' and less cohesivity.

\section{Nose and chin}

For projection of the nose and chin, it is necessary to overcome the high compression and shearing forces in these areas. Because the result of augmentation should be sustained for as long as possible, the filler should have high cohesivity and a high G'

\section{Nasolabial fold}

Two aspects need to be considered to improve nasolabial fold problems. First, the deep part should be considered because of the premaxillary depression. Therefore, a moderate to high G' filler is needed for the area to be volumized in order to avoid effects from compression and shear force. However, because it is also necessary to improve superficial wrinkles, a smooth filler should be selected with properties such as moderate cohesivity and a moderate G.' A high G' or large-particle filler is generally not used in this area due to the risk of visualization on the surface.

\section{CONCLUSIONS}

Many procedures in the field of plastic surgery use commercially produced filler materials. Therefore, we must comprehend the characteristics of filler materials, in addition to the need to enhance our skills as surgeons. Since the behavior of filler materials is a major factor contributing to good results, we have to comprehend their rheology_or in simple terms, their properties in terms of deformation and flow. Although rheology is a very difficult subject to understand thoroughly, it can be kept in mind that rheologic characteristics are determined by viscoelasticity and cohesivity, as expressed by various parameters.

Facial filler injections must take into account the various dynamics in each part of the face. Therefore, it is necessary to use fillers with various characteristics. Having a better comprehension of the rheologic properties of fillers will thus help clinicians to select the most appropriate filter to enhance clinical results.

\section{NOTES}

\section{Conflict of interest}

No potential conflict of interest relevant to this article was reported.

\section{ORCID}

Moon Seop Choi https://orcid.org/0000-0002-2307-5115

\section{REFERENCES}

1. Kontis TC. Contemporary review of injectable facial fillers. JAMA Facial Plast Surg 2013;15:58-64.

2. Cavallini M, Gazzola R, Metalla M, et al. The role of hyaluronidase in the treatment of complications from hyaluronic acid dermal fillers. Aesthet Surg J 2013;33:1167-74.

3. Flynn TC, Sarazin D, Bezzola A, et al. Comparative histology of intradermal implantation of mono and biphasic hyaluronic acid fillers. Dermatol Surg 2011;37:637-43.

4. Nast A, Reytan N, Hartmann V, et al. Efficacy and durability of two hyaluronic acid-based fillers in the correction of nasolabial folds: results of a prospective, randomized, doubleblind, actively controlled clinical pilot study. Dermatol Surg 2011;37:768-75.

5. Kablik J, Monheit GD, Yu L, et al. Comparative physical properties of hyaluronic acid dermal fillers. Dermatol Surg 2009;35 Suppl1:302-12.

6. Michaud T. Rheology of hyaluronic acid and dynamic facial rejuvenation: topographical specificities. J Cosmet Dermatol 2018; 17:736-43. 
7. Pierre S, Liew S, Bernardin A. Basics of dermal filler rheology. Dermatol Surg 2015;41 Suppl 1:S120-6.

8. Tezel A, Fredrickson GH. The science of hyaluronic acid dermal fillers. J Cosmet Laser Ther 2008; 10:35-42.

9. Sundaram H, Rohrich RJ, Liew S, et al. Cohesivity of hyaluronic acid fillers: development and clinical implications of a novel assay, pilot validation with a five-point grading scale, and evaluation of six U.S. Food and Drug Administrationapproved fillers. Plast Reconstr Surg 2015;136:678-86.

10. Stocks D, Sundaram H, Michaels J, et al. Rheological evaluation of the physical properties of hyaluronic acid dermal fillers. J Drugs Dermatol 2011;10:974-80.

11. Borrell M, Leslie DB, Tezel A. Lift capabilities of hyaluronic acid fillers. J Cosmet Laser Ther 2011;13:21-7. 